\title{
Risco e proteção de estudantes durante os anos finais do ensino fundamental
}

\author{
Ana Maria Nunes El Achkar \\ Universidade Salgado de Oliveira - Niterói - RJ - Faculdade CNEC Itaboraí -Rio de Janeiro - RJ - Brasil \\ Vanessa Barbosa Romera Leme \\ Universidade do Estado do Rio de Janeiro - Rio de Janeiro - RJ - Brasil \\ Adriana Benevides Soares \\ Universidade Salgado de Oliveira - Niterói - RJ - Universidade do Estado do Rio de Janeiro - Rio de Janeiro \\ $-R J-$ Brasil \\ Maria Angela Mattar Yunes \\ Universidade La Salle - Canoas - RS - Universidade Salgado de Oliveira - Rio de Janeiro - Brasil
}

\begin{abstract}
Resumo
O estudo comparou alunos com baixo, médio e alto desempenho escolar em relação aos fatores de risco: reprovação escolar e violência intra e extrafamiliar e de proteção: habilidades sociais, autoeficácia e apoio social. Participaram 400 estudantes $(M=13,6$ anos, $D P=1,22)$ que frequentavam os três últimos anos do Ensino Fundamental de escolas do Estado do Rio de Janeiro. Os alunos responderam ao Questionário de informações demográficas, Inventário de Habilidades Sociais para Adolescentes, Escala de Percepção de Apoio Social e Escala de Autoeficácia. Os resultados indicaram que alunos com baixo desempenho escolar apresentaram mais fatores de risco e menos fatores de proteção quando comparados aos alunos com médio e alto desempenho escolar. Não foram encontradas diferenças nas habilidades sociais de Assertividade, Abordagem afetiva e Desenvoltura social entre grupos. O estudo discute influências de variáveis pessoais e de contexto dos alunos para o desempenho escolar no final do Ensino Fundamental.
\end{abstract}

Palavras-chave: Resiliência; rendimento escolar; ensino fundamental.

\section{Risk and protection of students during the final years of elementary school}

\begin{abstract}
The study compared students with low, medium and high school performance in relation to risk factors: school disapproval and intra and extrafamiliar violence and protection: social skills, self efficacy and social support. A total of 400 students $(M=13.6$ years, $S D=1.22)$ participated in the last three years of elementary school education in the State of Rio de Janeiro. Students answered the Demographic Information Questionnaire, Social Skills Inventory for Adolescents, Social Support Perception Scale, and Self-efficacy Scale. The results indicated that students with low school performance had more risk factors and less protection factors when compared to students with medium and high school performance. No differences were found in the social skills of Assertiveness, Affective Approach and Social Development between groups. The study discusses influences of personal variables and context of students for school performance at the end of Elementary School.
\end{abstract}

Keywords: Resilience; school performance; elementary school.

\section{Riesgo y protección de estudiantes durante los años finales de la enseñanza primaria}

\section{Resumen}

En el estudio se comparó alumnos con bajo, mediano y alto desempeño escolar en relación a los factores de riesgo: repetición escolar y violencia dentro y fuera de la familia y de protección: habilidades sociales, autoeficacia y apoyo social. Participaron 400 estudiantes $(M=13,6$ años, $D P=$ $1,22)$ que frecuentaban los tres últimos cursos de la Enseñanza Primaria en escuelas del Estado de Rio de Janeiro. Los alumnos respondieron al Cuestionario de informaciones demográficas, Inventario de Habilidades Sociales para Adolescentes, Escala de Percepción de Apoyo Social y Escala de Autoeficacia. Los resultados indicaron que alumnos con bajo desempeño escolar presentaron más factores de riesgo y menos factores de protección cuando comparados a los alumnos con mediano y alto desempeño escolar. No se encontraron diferencias en las habilidades sociales de Asertividad, Abordaje afectivo y Desarrollo social entre grupos. En el estudio se discute influencias de variables personales y de contexto de los alumnos para el desempeño escolar en el final de la Enseñanza Primaria.

Palabras clave: Resiliencia; rendimiento escolar; enseñanza primaria. 


\section{Introdução}

No Brasil, dados do Índice da Educação Básica (Ministério da Educação, 2013) indicam que nos últimos anos do Ensino Fundamental (E.F.) há uma taxa de reprovação de $11,3 \%$, ou seja, aproximadamente 1.504 .238 estudantes não alcançam as exigências de aproveitamento ou frequência na escola. Conforme dados do Censo Escolar do Instituto Nacional de Estudos e Pesquisas Educacionais Anísio Teixeira (Inep, 2013), a percentagem de abandono neste mesmo ciclo está calculada em 3,6\%, o que corresponde a um total aproximado de 473.435 estudantes que abandonaram a escola durante o ano de 2013. Tais informações sinalizam um resultado negativo no rendimento acadêmico e apontam que a evasão se dá, sobretudo, durante os últimos anos do E.F.. Assim, verifica-se que esse momento da trajetória escolar merece ser priorizado em investigações.

Nesse âmbito destacam-se os estudiosos que investigam processos de resiliência e os resultados escolares positivos (Fletcher \& Sarkar, 2013; Zolkoski \& Bullock, 2012). Conforme Masten (2014, p.5), a resiliência pode ser definida como "a capacidade de um sistema dinâmico se adaptar de forma bem-sucedida e positiva às perturbações que ameaçam o seu funcionamento, a sua viabilidade ou o seu desenvolvimento". Estudar processos de resiliência implica em contemplar a operacionalização de conceitos de risco, proteção e de indicadores de ajustamento. Fatores de risco são compreendidos como os processos ambientais ou individuais que incidem sobre as vulnerabilidades da pessoa e podem desencadear resultados e influências negativas no desenvolvimento (Zolkosk \& Bullok, 2012). Os fatores de proteção funcionam de modo a diminuir, amenizar ou evitar o impacto dos riscos (Zolkosk \& Bullok, 2012). Esses possibilitam que os indivíduos a eles expostos busquem alternativas e soluções para as adversidades com as quais se defrontam (Amparo, Galvão, Alves, Brasil, \& Koller, 2008; Zolkosk \& Bullok, 2012). Conforme Coimbra e Fontaine (2015), resiliência e adaptação ou indicadores/critérios de ajustamento são termos diferentes e não devem ser entendidos como sinônimos. Segundo as autoras, alguns indicadores, como o bom desempenho escolar pode muitas vezes ser resultado de processos de resiliência e ser influenciado por fatores socioculturais, variando ao longo do ciclo de vida de cada pessoa.

$\mathrm{Na}$ adolescência o desempenho escolar é um importante indicador de ajustamento e se refere à capacidade de o aluno expressar o que aprendeu e adquiriu como conhecimento escolar no decorrer do processo de ensino-aprendizagem (Jacomini, 2004). Segundo D'Abreu e Marturano (2010), o baixo desempenho escolar ocorre quando o aluno apresenta, em notas ou tarefas, um resultado abaixo do nível esperado para a sua idade, traduzido por habilidades e potencial de um indivíduo. Conforme as autoras, variáveis pessoais e contextuais afetam o desempenho escolar e, desse modo, têm sido o foco, tanto de pesquisadores como de educadores que buscam identificar tais variáveis para planejar e desenvolver intervenções com os alunos com história de "insucesso" escolar. De acordo com Nunes, Pontes, Silva e Dell'Aglio (2014), os alunos com melhor desempenho escolar têm menos chance de repetência e evasão. Já os alunos com baixo desempenho escolar e menor percepção de apoio tendem a abandonar a escola (Amparo \& cols., 2008).

A resiliência é um processo dinâmico e contextual (Masten, 2014; Juliano \& Yunes, 2014) e, por esse motivo, torna-se necessário considerar em sua análise, o contexto sociocultural e as práticas econômicas e políticas, bem como os obstáculos e desafios com os quais os indivíduos sentem e enfrentam as adversidades presentes em seus contextos. De acordo com Masten (2014), fatores de risco e de proteção operam em diferentes domínios ecológicos, incluindo o grupo de pares, família, escola, vizinhança que influenciam o desenvolvimento. Neste sentido, a família, a vizinhança e a escola são compreendidos como os contextos mais importantes para os alunos e onde ocorrem as interações entre estes e os membros da família, pares e professores (Bronfenbrenner, 2002). Dependendo da qualidade das relações interpessoais estabelecidas naqueles ambientes, verifica-se tanto a promoção de saúde física e mental, isto é, de processos de resiliência, quanto o surgimento de dificuldades socioemocionais nos alunos. Desse modo, o presente estudo focaliza o indicador de ajustamento denominado desempenho escolar e sua interrelação com fatores de risco e de proteção presentes nos contextos mais importantes de alunos dos últimos anos do E.F.

\section{Fatores de risco: reprovação escolar e exposição à violência no contexto familiar e na comunidade}

Estudos indicam que a reprovação é o resultado de processos multideterminados que incluem fatores associados às características dos alunos e de suas famílias, das práticas escolares, da comunidade e de aspectos sociais, econômicos e políticos (Dazzani, Cunha, Luttigards, Zucoloto, \& Santos, 2014). Ademais, é uma manifestação de fracasso escolar que culmina em consequências adversas ao desenvolvimento socioafetivo e escolar dos alunos (Ingul, Klockner, Silverman, \& Nordahl, 2012). O estudo de Nunes e cols. (2014) evidenciou que apesar de apresentarem percepção positiva da escola, um índice significativo de estudantes, sobretudo da educação pública, já experimentou ser reprovado em um dado momento de seu percurso escolar. A conclusão é que o baixo desempenho acadêmico, por dificuldades no processo educativo, pode ocasionar o desinteresse do aluno pelo estudo, levando à reprovação e até mesmo ao abandono. Em consequência, tal fenômeno pode vir a constituir-se posteriormente em risco ao desenvolvimento humano (Ingul \& cols., 2012). Desse modo, a reprovação escolar pode ser um fator de risco, pois afeta a autoestima dos alunos, tornando-os mais fragilizados e propensos a abandonarem a escola (Ingul \& cols., 2012; Zolkosk \& Bullock, 2012).

A violência na família é outro fator de risco frequentemente investigado em crianças e adolescentes (Pacheco, Irigaray, Werlang, Nunes, \& Argimon, 2014). De uma forma 
generalizada, adolescentes vítimas de maus tratos na família demonstram rupturas em suas relações com os pares e apresentam prejuízos no desempenho escolar (Pacheco \& cols., 2014). Vítimas juvenis que sofrem violência intrafamiliar tendem a ter níveis de aproveitamento mais baixos na escola em comparação com aqueles que não foram maltratadas (Pacheco \& cols., 2014).

Segundo pesquisas, adolescentes expostos à violência física para além do ambiente da família intitulada violência extrafamiliar, também apresentam uma performance educacional "pobre" (Hammig \& Jozkowski, 2013, Pasian \& cols., 2014). Para Kokkinos e Kipritsi (2012), os alunos podem abandonar a escola por serem vítimas de violência e os problemas podem não se dissipar com a idade e progressão do ensino e mudança dos ciclos escolares (Hammig \& Jozkowski, 2013). Riscos contextuais persistentes sem a presença de fatores protetivos podem dificultar oportunidades de superação em momentos posteriores do curso de vida.

\section{Fatores de proteção dos alunos e de seus contextos: habilidades sociais, crenças de autoeficácia e apoio social}

Diante dos fatores de risco, como a reprovação escolar e a exposição à violência, as habilidades sociais e as crenças de autoeficácia têm sido identificadas como fatores de proteção dos alunos (Marturano \& Pizato, 2015; Zolkosk \& Bullok, 2012). Tais fatores são atrelados à percepção de apoio social da família, de professores e da comunidade como elementos de proteção de seus contextos, assim como são associados a bons resultados escolares (Juliano \& Yunes, 2014).

Habilidades sociais são compreendidas como um conjunto de comportamentos de um indivíduo aceitáveis socialmente dentro de um contexto, de uma cultura e de um tempo histórico que contribuem para a competência social e promovem relações interpessoais saudáveis (Del Prette \& Del Prette, 2005). O contexto escolar requer diversas habilidades interpessoais que devem ser apresentadas pelos alunos e que podem influenciar no seu desempenho escolar (Lopes, Del Prette, \& Del Prette, 2013). Estudos indicam que alunos com mais habilidades sociais apresentam melhor desempenho escolar (Feitosa, Del Prette, \& Del Prette, 2009; Marturano \& Pizato, 2015). Assim, as habilidades sociais podem funcionar como fatores de proteção para adolescentes com dificuldades socioemocionais e comportamentais.

De acordo com Bandura (2008), a autoeficácia refere-se às crenças individuais a respeito das próprias capacidades de organização e execução de determinadas ações no intuito de alcançar um resultado determinado. Tais crenças podem influenciar as escolhas que as pessoas fazem e os cursos de ação que prosseguem. Além disso, podem ajudar a determinar o esforço que as pessoas irão gastar em uma atividade, quanto tempo deverão perseverar quando confrontados aos obstáculos e seus processos de resiliência em face de situações adversas (Caprara, Vecchione, Alessandri, Gerbino, \& Barbaranelli, 2011). Desse modo, as crenças de autoeficácia exercem uma notável influência na aprendizagem, pois sustentam o esforço, a persistência e as aspirações dos alunos, levando a resultados escolares satisfatórios (Caprara \& cols., 2011).

Nessa direção, estudos (Oliveira \& Soares, 2011; Zuffiano \& cols., 2013) demonstraram que alunos com bom desempenho escolar apresentam maiores níveis de crenças de autoeficácia, tendo estas, portanto, alto valor explicativo para desempenho nas tarefas escolares. O bom desempenho escolar dos estudantes, além de sofrer influências de variáveis intrapessoais, é também afetado por relações interpessoais presentes nos contextos mais amplos, como na família, na escola e na comunidade (Juliano \& Yunes, 2014). Conforme Mackinnon (2012), a compreensão do indivíduo inserido em seu contexto ecológico permite uma análise mais vasta sobre aquele em sua vertente mais favorável e saudável, identificando os fatores e processos que alavancam o seu desenvolvimento positivo.

O apoio social é compreendido a partir das funções que um grupo de pessoas exerce em favor de um indivíduo que está imerso em situações desafiadoras e de risco (Juliano \& Yunes, 2014). Podem ser caracterizadas por presenças de membros da família, de amigos e/ou vizinhos ou até mesmo pessoas desconhecidas que desempenham papel de ajuda e suporte em momentos considerados críticos (Squassioni \& Matsukura, 2014). Antunes e Fontaine (2008) afirmam que a percepção do apoio social age como facilitador da adaptação do indivíduo frente às mudanças durante as transições dos variados níveis de escolarização. Resulta, portanto, em sensação de segurança e bem-estar no contexto escolar. Nesta perspectiva, os anos finais do E.F. representam uma fase delicada em que os alunos começam a escolher qual percurso acadêmico seguirão (Mackinnon, 2012). Dessa forma, o papel protetivo desempenhado por famílias, amigos e professores é capaz de colaborar com os adolescentes na definição e estabelecimento de suas metas e é importante preditor do bom desempenho acadêmico (Zuffiano \& cols., 2013).

A rede de apoio favorece processos de resiliência em crianças, adolescentes e jovens por trazer-lhes maior confiança em si mesmos, gerando fatores de proteção percebidos em seus contextos (Amparo \& cols., 2008). O apoio social funciona interagindo sobre fatores de risco, possibilitando outras escolhas e versatilidade de opções para solucionar as situações problema concernentes ao dia-a-dia em âmbito social e emocional, criando uma situação de empoderamento nos indivíduos (Amparo \& cols., 2008; Juliano \& Yunes, 2014).

A partir dessas considerações, estudos têm mostrado evidências consistentes para correlações percebidas entre suportes sociais e bom desempenho escolar em adolescentes (Mackinnon, 2012; Zuffiano \& cols., 2013). O apoio social dos professores soma-se aos apoios sociais da família e dos pares. Ao constatarem o apoio de seus professores esses alunos apresentam melhores notas do que aqueles que não denotam a percepção desse apoio (Ahmed, Minnaert, Werf, \& Kuyper, 2010). Assim, durante as duas últimas décadas, a escola e a formação da relação afetiva entre professor-aluno têm sido apontadas como importante e significativa rede de 
apoio social (Machado, Yunes, \& Silva, 2014; Nunes \& cols., 2014; Roorda \& Koomen, 2011; Yunes, Pietro, Silveira, Juliano, \& Garcia, 2015). Contudo, outros ambientes externos a escola, também auxiliam na percepção de apoio aos estudantes, contribuindo para processos de resiliência e para um bom desempenho escolar (Amparo \& cols., 2008). Estudos mostram que ao longo da vida, os indivíduos são cercados por redes sociais tecidas por uma variedade de outros significativos que incluem pessoas da comunidade que prestam imensurável apoio social (Juliano \& Yunes, 2014; Sterrett, Jones, McKee, \& Kincaid, 2011).

A partir da revisão de literatura foi possível verificar que variáveis pessoais e contextuais podem influenciar o desempenho escolar dos alunos durante os anos finais do E.F. Essas variáveis, quando analisadas sob o enfoque dos processos de resiliência, permitem visualizar as interfaces de fatores de risco e de proteção presentes no âmbito individual e contextual. Contudo, não foi encontrado nenhum estudo que articulasse tais riscos e proteção ao desempenho escolar de alunos ao final da educação básica. Poder-se-ia afirmar que esta é uma lacuna no conhecimento acerca desta etapa da trajetória escolar. Assim, este estudo teve como objetivo contribuir para a compreensão dessa fase a partir de uma investigação que busca comparar alunos dos anos finais do E.F. com baixo, médio e alto desempenho escolar em relação aos fatores de risco: reprovação escolar e violência intra e extrafamiliar e os fatores de proteção: habilidades sociais, autoeficácia e apoio social.

\section{Método}

\section{Participantes}

Foi realizado um estudo transversal com comparação de grupos. A amostra foi selecionada por conveniência e composta por 400 estudantes, com idades entre 11 a 17 anos $(M=13,6$ anos, $D P=1,22)$, sendo que 220 (55\%) meninas e $180(45 \%)$ meninos que frequentavam o $7^{\circ}$ ano ( $\mathrm{n}$ $=135,33,8 \%), 8^{\circ}$ ano $(n=145,36,3 \%)$ e $9^{\circ}$ ano $(n=120$, $30,0 \%$ ) do E.F., provenientes de quatro escolas, sendo duas públicas e duas particulares, situadas no Estado do Rio de Janeiro. Os estudantes encontravam-se em sua maioria entre as classes socioeconômicas B2 e B1(53,8\%), 26\% na classe $A 2,13,5 \%$ na classe $C 1,5,0 \%$ na classe $A 1,1,3 \%$ na classe C2 e 0,3\% na classe D (Associação Brasileira e Empresas de Pesquisa [ABEP], 2013).

Os participantes do estudo foram divididos em três grupos classificados por: alto desempenho escolar; médio desempenho escolar; baixo desempenho escolar. Para essa classificação foram utilizadas as notas de todas as disciplinas para o cálculo da média final denominada de "média escolar" (varia de 0,0 a 10,0), adquiridas por meio de Atas de Resultados Finais com as coordenadoras pedagógicas das escolas participantes do estudo. A partir das médias escolares de todos os alunos, foram obtidos os quartis por meio do programa Statistical Package for the Social Sciences for Windows (SPSS, versão 22.0), de modo que tais valores foram divididos em quatro intervalos: um intervalo com $25 \%$ dos valores mais baixos; dois intervalos formados por $25 \%$ dos valores intermediários; um intervalo com $25 \%$ dos valores mais altos. Assim, o critério para a classificação em um dos três grupos foi o intervalo em que esse valor se encontrava. No primeiro quartil (média escolar menor que 5,70 ), os alunos foram classificados como baixo desempenho escolar $(n=87$ ). No segundo e terceiro quartis (média escolar entre 5,70 e menor que 7,80 ), os alunos foram classificados como médio desempenho escolar $(\mathrm{n}=211)$. No quarto quartil (média escolar maior que 7,80 ), os estudantes foram classificados com alto desempenho escolar $(n=102)$.

\section{Instrumentos}

Questionário com informações demográficas dos alunos e Questionário Critério de Classificação Econômica Brasil. O primeiro foi elaborado para este investigar informações sociais e demográficas, divididos em três partes: (a) informações gerais de aplicação; (b) informações sobre os participantes (nome, idade, sexo e reprovação escolar - se já tinha sido reprovado de ano e quantas vezes); (c) informações sobre a família (número e indicação de quem residia com os alunos). Foi também empregado o Questionário Critério de Classificação Econômica Brasil - CCBB (ABEP, 2013) que avalia o nível socioeconômico e permite a estratificação das famílias, em ordem decrescente de poder aquisitivo e nível de escolaridade, em cinco classes: $A$ (subdividida em A1 e A2), B (subdividida em B1 e B2), C, D e E.

Inventário de Habilidades Sociais para Adolescentes (IHSA-Del-Prette). É um instrumento desenvolvido por Z. Del Prette e Del Prette (2009) que avalia as habilidades sociais de adolescentes a partir dos seus autorrelatos sobre situações cotidianas. Apresenta 38 itens que contemplam diferentes habilidades divididas em seis fatores: Empatia; Autocontrole; Civilidade; Assertividade; Abordagem Afetiva; e Desenvoltura Social. As respostas estão dispostas em uma escala do tipo Likert que varia de 0 (nunca) a 4 (sempre). No presente estudos foram encontrados os seguintes índices de consistência interna: $\alpha=0,84$ para a escala total e de 0,48 a 0,74 para os fatores de frequência.

Escala de Percepção de Apoio Social (EPAS). É um instrumento desenvolvido por Vaux e cols. (1986) para o contexto americano para examinar a percepção de apoio social de crianças e adolescentes em relação à família e aos amigos (Social Support Appraisal - SSA). A escala foi validada para a população de adolescentes brasileiros por Squassoni e Matsukura (2014). É composta por 30 itens disposto numa escala tipo Likert de 1 (Discordo totalmente) a 6 (Concordo totalmente) que contemplam quatro fatores: (1) Percepção de apoio social da família; (2) Percepção de apoio social dos amigos; (3) Percepção de apoio social dos professores; (4) e Percepção de apoio social dos outros em geral. No presente estudo foram encontrados os seguintes 
índices de consistência interna: $\alpha=0,91$ para a escala total e de 0,80 a 0,89 para os fatores.

Escala de Autoeficácia Generalizada (EAG). É originária da Alemanha (Schwarzer \& Jerusalem, 1995) e foi adaptada para diferentes culturas para identificar as crenças de autoeficácia diante de situações difíceis de participantes de diferentes origens socioeconômicas e idades, incluindo adolescentes. No Brasil, foi adaptada para uma amostra de adolescentes por Leme, Coimbra, Gato, Fontaine e Del Prette (2013). É constituído por 10 itens distribuídos numa escala tipo Likert de 1 (Discordo Totalmente) a 4 (Concordo Totalmente). No presente estudo foi encontrado o seguinte índice de consistência interna para a escala: $\alpha=0,79$.

Questionário da Juventude Brasileira (QJBra, Versão Fase II). É um instrumento utilizado para examinar fatores de risco e proteção de adolescentes e jovens (14 a 24 anos) de diversos contextos e nível socioeconômico baixo. O questionário foi desenvolvido Dell'Aglio, Koller, Cerqueira-Santos e Colaço (2011) e contém 77 questões de múltipla escolha. No presente estudo, para a coleta dos dados a respeito dos Fatores de Risco, selecionaram-se as questões 31 (violência intrafamiliar), 62 (violência extrafamiliar), com escala nominal (presença ou ausência) e a questão 68, para avaliar a rede de apoio da comunidade, contendo 6 itens em escala tipo Likert de 1 (Nunca) a 5 (Sempre). Na presente pesquisa foi encontrado o seguinte índice de consistência interna para a questão 68: apoio social da comunidade, $\alpha=0,80$.

\section{Procedimentos}

Coleta de dados. A pesquisa foi aprovada, de acordo com o Parecer $n^{\circ}$ 921.458/2014 - CAAE: 39701814.5.0000.5289, pelo Comitê de Ética em Pesquisa da Universidade. Após a entrega do Termo de Consentimento Livre e Esclarecido (TCLE), assinado pelos responsáveis legais dos alunos, a coleta de dados foi realizada, de forma coletiva nas salas de aula, no horário que foi combinado previamente com os professores. No início de cada aplicação (duração média de 50 minutos) foi apresentado aos alunos o objetivo da pesquisa, a garantia de confidencialidade dos dados coletados e reiterado o caráter voluntário da sua participação.

Análise de dados. A análise de dados foi executada com o software Statistical Package for the Social Sciences for Windows (SPSS, versão 22.0). Foram realizadas análises descritivas e inferenciais. Primeiramente, foi confirmado o pressuposto de normalidade e homogeneidade das variâncias. Na sequência, para comparar grupos de alunos foram realizadas análises de variância a um fator (Anova one-way), com teste Post Hoc Tukey.

\section{Resultados}

A Tabela 1 apresenta os resultados das comparações dos fatores de risco e proteção entre os alunos com baixo, médio e alto desempenho escolar do $7^{\circ}, 8^{\circ}$ e $9^{\circ}$ ano do E.F.
A Tabela 1 indica que 19,8\% dos estudantes haviam reprovado de ano, $45,5 \%$ informaram ter sofrido algum tipo de violência no âmbito familiar e $40 \%$ fora da família. Os resultados revelam que os alunos dos grupos com baixo e médio desempenho escolar apresentam mais reprovação escolar e são mais expostos à violência intra e extrafamiliar do que os alunos com alto desempenho escolar.

No que diz respeito aos fatores de proteção, os dados mostram com diferenças significativas, que os estudantes do grupo com alto desempenho escolar apresentam mais habilidades sociais de Empatia, Autocontrole, Civilidade e no total das habilidades sociais quando comparados aos alunos dos grupos com baixo e médio desempenho escolar. Para essas habilidades sociais e no total, não houve diferenças estatísticas entre os grupos com médio e baixo desempenho escolar.

Os dados indicam que não houve diferença significativa entre os três grupos em relação às habilidades sociais de Assertividade, Abordagem afetiva e Desenvoltura social. Os resultados das análises demonstram que os grupos apresentam diferenças significativas entre si em relação às crenças de autoeficácia e à percepção de apoio social da família, dos outros, no total e da comunidade. Com relação à percepção de apoio social dos amigos e dos professores, os grupos com baixo e médio desempenho escolar são semeIhantes. Os alunos com alto desempenho escolar apresentam mais percepção de apoio dos amigos e da família quando comparados aos alunos com baixo e médio desempenho.

\section{Discussão}

No que se refere aos fatores de risco, os dados indicaram que os alunos com baixo e médio desempenho escolar reprovaram mais vezes que os alunos com alto desempenho. Esses resultados estão de acordo com alguns estudos (Ingul \& cols., 2012; Nunes \& cols., 2012; Zolkosk \& Bullock, 2012) que evidenciaram que a reprovação escolar pode impactar negativamente na autoestima do aluno, tornando-o mais fragilizado a ponto de levá-los a abandonar a escola, o que transcende o fenômeno da reprovação e amplia suas consequências negativas na escolaridade dos alunos desta faixa etária. Nunes e cols. (2014) afirmam que, por isso, a reprovação escolar aumenta a chance de os estudantes desistirem da escola e deve ser considerada como importante elemento de risco ao desenvolvimento saudável.

Além de apresentar maior número de reprovações, os alunos com baixo e médio desempenho escolar também indicaram ter sido mais expostos à violência na família e fora de casa. Corroborando com esses resultados, pesquisas (Hammig \& Jozkowski, 2013; Pacheco \& cols., 2014) evidenciaram que alunos que passam por violência, seja dentro no contexto familiar ou na comunidade, podem apresentar desempenho escolar mais baixo, distúrbios comportamentais e/ou patologias físicas e psicológicas. Parece, portanto, que alunos com desempenho escolar abaixo do esperado apresentam em seus contextos um acúmulo de fatores de 
Tabela 1. Fatores de Risco e Proteção: Resultados das Comparações entre os Alunos dos Grupos com Baixo, Médio e Alto Desempenho Escolar.

\begin{tabular}{|c|c|c|c|c|}
\hline \multirow[b]{2}{*}{ Variáveis } & \multicolumn{4}{|c|}{ Estudantes } \\
\hline & $\begin{array}{l}\text { Grupo baixo } \\
\mathrm{DE}(\mathrm{n}=87)\end{array}$ & $\begin{array}{l}\text { Grupo médio DE } \\
\qquad(n=211)\end{array}$ & $\begin{array}{l}\text { Grupo alto } \\
D E(n=102)\end{array}$ & $\begin{array}{c}\text { Total } \\
(\mathrm{N}=400)\end{array}$ \\
\hline Fatores de Risco & $F(\%)$ & $F(\%)$ & $F(\%)$ & $F(\%)$ \\
\hline \multicolumn{5}{|l|}{ Reprovação escolar } \\
\hline Não & $57(17,8 \%)$ & $172(53,5 \%)$ & $92(28,7 \%)$ & $321(80,3 \%)$ \\
\hline Sim & $30(37,9 \%)$ & $39(49,4 \%)$ & $10(12,7 \%)$ & $79(19,8 \%)$ \\
\hline \multicolumn{5}{|l|}{$\begin{array}{l}\text { Exposição à violência na } \\
\text { família }\end{array}$} \\
\hline Nunca & $29(13,9 \%)$ & $108(51,9 \%)$ & $81(34,2 \%)$ & $218(54,5 \%)$ \\
\hline Já aconteceu & $58(31,8 \%)$ & $103(56,6 \%)$ & $21(11,6 \%)$ & $182(45,5 \%)$ \\
\hline \multicolumn{5}{|c|}{$\begin{array}{l}\text { Exposição à violência fora de } \\
\text { casa }\end{array}$} \\
\hline Nunca & $42(17,5 \%)$ & $121(50,4 \%)$ & $77(32,1 \%)$ & $240(60 \%)$ \\
\hline Já aconteceu & $45(28,3 \%)$ & $90(56,2 \%)$ & $25(15,6 \%)$ & $160(40 \%)$ \\
\hline Fatores de proteção & $M(D P)$ & $M(D P)$ & $M(D P)$ & $F$ \\
\hline \multicolumn{5}{|l|}{ Habilidades Sociais } \\
\hline Empatia & $24,91(6,89)^{a}$ & $25,71(6,05)^{a}$ & $28,49(5,75)^{b}$ & $9,58^{*}$ \\
\hline Autocontrole & $14,07(5,64)^{a}$ & $14,67(6,01)^{a}$ & $17,40(6,35)^{b}$ & $9,14^{*}$ \\
\hline Civilidade & $17,56(4,44)^{a}$ & $18,19(3,96)^{a}$ & $19,55(3,71)^{\mathrm{b}}$ & $6,35^{*}$ \\
\hline Assertividade & $19,40(5,11)^{a}$ & $19,86(4,71)^{a}$ & $20,09(4,60)^{a}$ & 0,50 \\
\hline Abordagem Afetiva & $10,84(4,67)^{a}$ & $11,15(4,47)^{a}$ & $12,12(4,71)^{a}$ & 2,16 \\
\hline Desenvoltura Social & $10,38(4,08)^{a}$ & $10,67(3,74)^{a}$ & $11,60(4,02)^{a}$ & 2,75 \\
\hline Total & $97,16(22,79)^{a}$ & $\begin{array}{c}100,25 \\
(19,86)^{a}\end{array}$ & $\begin{array}{c}109,25 \\
(19,93)^{b}\end{array}$ & $9,50^{*}$ \\
\hline \multicolumn{5}{|l|}{ Autoeficácia } \\
\hline Total & $27,21(5,38)^{a}$ & $28,85(5,22)^{b}$ & $30,87(4,37)^{c}$ & $12,54^{*}$ \\
\hline \multicolumn{5}{|l|}{ Percepção de Apoio Social } \\
\hline Família & $38,36(9,68)^{a}$ & $41,38(7,73)^{b}$ & $44,63(4,83)^{\mathrm{c}}$ & $16,05^{*}$ \\
\hline Amigos & $33,11(7,15)^{a}$ & $34,55(6,31)^{a}$ & $36,98(5,05)^{\mathrm{b}}$ & $9,62^{*}$ \\
\hline Professores & $26,56(7,39)^{a}$ & $26,91(7,97)^{a}$ & $32,00(6,52)^{b}$ & $18,26^{*}$ \\
\hline Outros & $32,40(8,46)^{a}$ & $36,28(6,72)^{\mathrm{b}}$ & $39,26(6,39)^{c}$ & $22,21^{*}$ \\
\hline Total & $135,18(27,17)^{\mathrm{a}}$ & $\begin{array}{c}144,01 \\
(21,76)^{b}\end{array}$ & $\begin{array}{c}158,12 \\
(18,51)^{c}\end{array}$ & $26,06^{*}$ \\
\hline \multicolumn{5}{|l|}{$\begin{array}{l}\text { Percepção de apoio da } \\
\text { Comunidade }\end{array}$} \\
\hline Total & $14,56(5,21)^{a}$ & $16,72(5,47)^{b}$ & $19,24(5,14)^{c}$ & $18,23^{*}$ \\
\hline
\end{tabular}

Nota. $\mathrm{N}=400$. DE: Desempenho Escolar. Letras diferentes indicam diferenças estatísticas significativas entre os grupos. Teste Post Hoc Tukey. ${ }^{*} p \leq 0,05$ 
risco que pode interferir no surgimento de processos de resiliência.

Em relação aos fatores de proteção, verificou-se que os alunos com baixo desempenho escolar apresentaram menos habilidades sociais de Empatia, Autocontrole, Civilidade, Desenvoltura social e total que os participantes com alto desempenho escolar. Esses dados vão ao encontro de outros estudos (Feitosa \& cols., 2009; Lopes \& cols., 2013; Marturano \& Pizato, 2015) que verificaram que alunos com déficits em habilidades sociais podem apresentar menor desempenho escolar. Porém, os dados indicaram que não houve diferença entre os estudantes com baixo, médio e alto desempenho no que se refere às habilidades sociais de Assertividade, Abordagem afetiva e Desenvoltura social, o que sugere que tais habilidades podem ser consideradas como protetoras principalmente para os alunos com baixo desempenho escolar. $Z$. Del Prette e Del Prette (2009) afirmam que a assertividade é a capacidade de o indivíduo expressar sentimentos positivos e negativos, discordar de opiniões entre outros, sem agredir ou manter-se passivo. Desse modo, pode-se sugerir que a assertividade dos estudantes pode vir a inibir condições adversas e potencializar o aproveitamento escolar, apresentando ganhos significativamente maiores para a aprendizagem, aquisição de novos conhecimentos e, por conseguinte, no desempenho escolar (Lopes \& cols., 2013).

Já a abordagem afetiva envolve, segundo Z. Del Prette e Del Prette (2009), a habilidade de se relacionar com outros indivíduos, de fazer e responder a perguntas de cunho pessoal, ser capaz de iniciar e manter uma conversa, fazer parte de equipes de trabalho escolar, relacionar-se de forma íntima quando houver interesse e ser capaz de expressar satisfação ou insatisfação como reação a diversas modalidades de carinho. Quando os estudantes apresentam essas habilidades, seus comportamentos tendem a ser mais ajustados e reduzem os efeitos do risco, fortalecendo suas competências de modo que as condições e agentes estressores sejam eliminados ou minimizados, levando-os a um melhor desempenho escolar (Z. Del Prette \& Del Prette, 2009).

Por fim, a habilidade social de Desenvoltura social envolve qualidade de fluência e capacidade de se comunicar de maneira desembaraçada e com desenvoltura (Z. Del Prette \& Del Prette, 2009). Tal habilidade pode possibilitar que, diante de situações de avaliação e julgamento, os alunos tenham mais facilidade em qualificar de forma adequada e eficaz como devem se comportar. Desse modo, pode-se dizer que o fato dos estudantes apresentarem essa habilidade social sugere que eles poderão traçar e cumprir metas claras e objetivas, mantendo e melhorando suas relações com os demais atores do cenário escolar, delimitando o equilíbrio entre as relações pré-estabelecidas de poder e mantendo e/ ou melhorando sua autoestima e sua autoeficácia (Soares, Poubel, \& Mello, 2009).

Contudo, os alunos com baixo desempenho escolar apresentaram menores níveis de autoeficácia que os alunos com médio e alto desempenho escolar. Assim, em seu conjunto de crenças e expectativas, estudantes com baixo desempenho escolar podem não se sentir capazes de realizar tarefas e atividades para concretizar objetivos e metas para o alcance de resultados desejados no que tange as atividades escolares educativas. Conforme alguns estudos (Caprara \& cols., 2011; Zuffiano \& cols., 2013), a falta de autoeficácia está relacionada com as fracas expectativas e com o insucesso escolar. Para Hoigaard, Kovac, Overby e Haugen (2015), níveis mais altos de crenças de autoeficácia são importantes para a consecução desejável de níveis elevados de alto desempenho escolar e afetam positivamente a autoestima. Nesse sentido, Bastianello, Pacico e Hutz (2014), sinalizam que os indivíduos com baixa autoestima tendem a ter ansiedade excessiva e dificuldade para tolerar a frustração causada pela não-realização de desejos, fornecendo respostas de enfrentamento mal adaptativas. A presença de um ambiente de aprendizagem orientado, com a presença de uma forte rede de apoio focado para a realização da tarefa, aparece como importante fator de proteção para o bom desempenho escolar (Bastianello \& cols., 2014; Hoigaardet \& cols., 2015).

No que se refere ao apoio social, os resultados indicaram que estudantes com baixo desempenho escolar perceberam menos apoio social dos familiares, de outras pessoas em geral e da comunidade quando comparados aos alunos com médio e alto desempenho escolar. De fato, de acordo com algumas pesquisas (Zuffiano \& cols., 2013; Amparo \& cols., 2008; Juliano \& Yunes, 2014) alunos com mais apoio social apresentam melhores resultados escolares, com maiores possibilidades de promoção de resiliência e empoderamento no ambiente escolar.

A percepção de apoio social é apontada pela literatura (Mackinnon, 2012; Squassioni \& Matsukura, 2014; Yunes \& cols., 2015) como um importante fator externo de proteção para adolescentes expostos à violência. Chamou a atenção que os alunos com baixo e médio desempenho escolar não apresentaram diferenças quanto à percepção de apoio social dos amigos e dos professores, embora essa percepção tenha sido menor em relação ao grupo com alto desempenho escolar. Pesquisas (Mackinnon, 2012; Roorda \& Koomen, 2011) têm evidenciado que além do apoio social dos membros da família, as relações com pares estão associadas positivamente com o bem-estar e bom desempenho escolar. Além disso, Roorda e Koomen (2011) indicaram em seu estudo que a percepção de apoio social dos professores está associada a resultados acadêmicos mais positivos. Professores sensíveis podem servir como "tutores de resiliência" e base segura a partir da qual os alunos podem explorar o ambiente escolar e se engajarem em atividades de aprendizagem (Machado \& cols., 2014; Nunes \& cols., 2014; Roorda \& Koomen, 2011; Yunes \& cols., 2015). Assim, considerando os dados do presente estudo, seria importante que futuras pesquisas de intervenção procurassem desenvolver habilidades sociais educativas e relacionais dos professores para que esses possam contribuir para fomentar e fortalecer o vínculo socioafetivo com seus alunos e entre eles. 


\section{Considerações finais}

A presente pesquisa contribuiu para a compreensão de como as influências das variáveis pessoais e do contexto dos alunos podem afetar os resultados acadêmicos nos anos finais do E.F. Uma das questões mais indefinidas entre os pesquisadores das ciências da Psicologia e da Educação refere-se ao "peso" do impacto de aspectos individuais na complexa interação com elementos sociais, culturais e ecológicos para o desenvolvimento humano. Este estudo evidenciou tais nuances e denota claramente a necessidade do investimento em pesquisas na comunidade que indiquem caminhos para intervenções positivas. Na busca incessante por essas respostas e soluções de promoção de resiliência em indivíduos, famílias e comunidades sublinha-se que é preciso investir em políticas públicas sociais que gerem mais oportunidades protetivas do que riscos e assim, maximizem as potencialidades individuais e comunitárias das nossas sociedades e culturas.

Algumas limitações do presente estudo devem ser consideradas. Em primeiro lugar, os resultados são específicos de uma determinada amostra que foi selecionada por conveniência a partir de algumas escolas públicas e privadas situadas no estado do Rio de Janeiro. Em segundo lugar, os dados também foram coletados a partir das percepções dos alunos e, desse modo, seria interessante que futuros estudos incluíssem informações coletadas com outros informantes, como pais e professores. Por fim, deve-se considerar que os grupos com baixo, médio e alto desempenho escolar foram formados por meio das médias escolares que são medidas não padronizadas de competências. Desse modo, se por um lado tais medidas oferecem dados contextualizados da realidade educacional das escolas pesquisas, por outro limitam a comparação com outros locais.

Levando-se em conta as limitações, os achados deste estudo ressaltam que os alunos com baixo desempenho escolar que frequentam os anos finais do E.F. são pouco pesquisados, o que suscita uma reflexão para as implicações da falta de dados a esse respeito. Estas implicações estão voltadas para a maior vulnerabilidade de alunos com baixo desempenho escolar quando comparados aos alunos com médio e alto desempenho, o que pode resultar em abandono escolar e processos de exclusão dos ambientes educativos. Entretanto, as habilidades sociais de assertividade, abordagem afetiva e desenvoltura social foram iguais para os três grupos. Isso sinaliza que recursos pessoais dos alunos com baixo/médio desempenho escolar podem ser mobilizados em futuros programas de intervenção que envolvam diferentes segmentos relacionais, tais como professores, pais e alunos.

\section{Referências}

Ahmed, W., Minnaert, A., Werf, G. V., \& Kuyper, H. (2010). Perceived social support and early adolescents' achievement: The mediational roles of motivational beliefs and emotions. Journal of Youth and Adolescence, 39(1), 36-46.
Amparo, D. M., Galvão, A. C. T., Alves, P. B., \& Koller, S. H. (2008). Adolescentes e jovens em situação de risco psicossocial: Redes de apoio social e fatores pessoais de proteção. Estudos de Psicologia, 13(2), 165-174.

Antunes, C.\& Fontaine, A. M. (2008). Percepção de apoio social na adolescência: Análise fatorial confirmatória da escala Social Support Appraisal. Paidéia, 15(32), 355-366.

Associação Brasileira de Empresas de Pesquisa [ABEP] (2013). Dados com base no Levantamento Socioeconômico de 2012. Recuperado: 26 jan. 2015. Disponível: http://www.abep.org/ criterio-brasil.

Bandura, A. A. (2008). A evolução da teoria social cognitiva. Em A. Bandura, R. G. Azzi, \& S. A. J. Polydoro (Orgs.), Teoria social cognitiva: Conceitos básicos (pp. 15-41). Porto Alegre: Artmed.

Bronfenbrenner, U. (2002). A ecologia do desenvolvimento humano: Experimentos naturais e planejados (M. A. V. Veronese, Trad.). Porto Alegre: Artmed (Trabalho original publicado em 1979).

Bastianello, M. R., Pacico, J. C., \& Hutz, C. S. (2014). Optimism, selfesteem and personality: Adaptation and validation of the Brazilian Version of the Revised Life Orientation Test (LOT-R). Psico-USF, 19(3), 523-531.

Caprara, G. V., Vecchione, M., Alessandri, G., Gerbino, M., \& Barbaranelli, C. (2011). The contribution of personality traits and self-efficacy beliefs to academic achievement: A longitudinal study. British Journal of Educational Psychology, 81, 78-96.

Coimbra, S. \& Fontaine, A. M. V. G. (2015). Resiliência e habilidades sociais: Reflexões conceituais e práticas para uma nova geração. Em Z. A. P. Del Prette, A. B. Soares, C. S. Pereira-Guizzo, M. F. Wagner, \& V. B. R. Leme (Orgs.), Habilidades sociais: Diálogos e intercâmbios sobre pesquisa e prática (pp. 186-220). Novo Hamburgo, RS: Sinopsys.

Dazzani, M. V. M., Cunha, E. O., Luttigards, P. M., Zucoloto, P. C S. V., \& Santos G. L. (2014). Queixa escolar: Uma revisão crítica da produção científica nacional. Psicologia Escolar e Educacional, 18(3), 421-428.

D'Abreu, L. C. F. \& Marturano, E. M. (2010). Associação entre comportamentos externalizantes e baixo desempenho escolar: Uma revisão de estudos prospectivos e longitudinais. Estudos de Psicologia, 15(1), 43-51.

Dell'Aglio, D. D., Koller, S. H., Cerqueira-Santos, E., \& Colaço, V. F. R. (2011). Revisando o Questionário da Juventude Brasileira: Uma nova proposta. Em D. Dell'Aglio \& S. Koller (Orgs.), Adolescência e Juventude: Vulnerabilidade e contextos de proteção (pp. 259270). São Paulo: Casa do Psicólogo.

Del Prette, Z. A. P. \& Del Prette, A. (2005). Psicologia das habilidades sociais na infância: Terapia e educação. Petrópolis: Vozes. 
Del Prette, Z. A. P. \& Del Prette, A. (2009). Inventário de Habilidades Sociais para adolescentes. (IHSA - Del-Prette): Manual de aplicação, apuração e interpretação. São Paulo: Casa do Psicólogo.

Feitosa, F. B., Matos, M. G., Del Prette, Z. A. P., \& Del Prette, A. (2009). Desempenho acadêmico e interpessoal em adolescentes portugueses. Psicologia em Estudo, 14(2), 259-266.

Fletcher, D. \& Sarkar, M. (2013). Psychological resilience: A review and critique of definitions, concepts and theory. European Psychologist, 18(1), 12-23.

Hammig, B. \& Jozkowski, K. (2013). Academic achievement, violent victimization, and bullying among U.S. high school students. Journal of Interpersonal Violence, 28(7), 1424-1436.

Hoigaard, R., Kovac, V.B., Overby, N.C., \& Haugen, T. (2015). Academic self-efficacy mediates the effects of school psychological climate on academic achievement. School Psychology Quarterly, 30(1), 64-74.

Ingul, M.J., Klockner, C.A., Silverman, W.K., \& Nordahl, H.M. (2012). Adolescent school absenteeism: Modelling social and individual risk factors. Child and Adolescent Mental Health, 17(2), 93-100.

Instituto Nacional de Estudos e Pesquisas Educacionais Anísio Teixeira [Inep] (2013). Censo escolar 2013. Brasília, DF: Inep. Recuperado: 23 jan. 2014. Disponível: http://portal.inep.gov.br/ basica-censo.

Jacomini, M.A. (2004). A escola e os educadores em tempo de ciclos e progressão continuada: Uma análise das experiências no estado de São Paulo. Educação e Pesquisa, 30(3), 401-418. Recuperado: 19 mar. 2014. Disponível: www.scielo.br/pdf/ep/v30n3/a02v30n3.pdf

Juliano, M.C.C. \& Yunes, M.A.M. (2014). Reflexões sobre rede de apoio social como mecanismo de proteção e promoção de resiliência. Ambiente \& Sociedade, 3(1), 135-154.

Kokkinos, C.M. \& Kipritsi, E. (2012). The relationship between bullying, victimization, trait emotional intelligence, self-efficacy and empathy among preadolescents. Social Psychology of Education, 15(1), 41-58.

Leme, V.B.R., Coimbra, S., Gato, J., Fontaine, A.M., \& Del Prette, Z.A.P. (2013). Confirmatory Factor Analysis of the Generalized Self-Efficacy Scale in Brazil and Portugal. Spanish Journal of Psychology, 16(E93), 1-11.

Lopes, D.C., Del Prette, Z.A.P., \& Del Prette,A. (2013). Recursos multimídia na promoção de habilidades sociais de crianças com baixo rendimento acadêmico. Psicologia: Reflexão e Crítica, 26(3), 451-458.

Machado, J.A., Yunes, M.A., \& Silva, G.F. (2014). A formação continuada de professores em serviço na perspectiva da abordagem ecológica do desenvolvimento humano. Contrapontos, 14(3), 512-526.
Mackinnon, S.P. (2012). Perceived social support and academic achievement: Cross-Lagged panel and bivariate growth curve analyses. Journal Youth Adolescence, 41(2), 474-485.

Marturano, E.M. \& Pizato, E.C.G. (2015). Preditores do Desempenho Escolar no $5^{\circ}$ Ano do Ensino Fundamental. Psico46(1), 16-24.

Masten, A.S. (2014). Global Perspectives on Resilience in Children and Youth. Child Development, 85(1), 6-20.

Ministério da Educação (2013). Índice da Educação Básica. Brasília: Ideb. Recuperado: 10 jun. 2014. Disponível: http://ideb.inep.gov. $\mathrm{br} /$ resultado

Nunes, T.G.R., Pontes, F.A.R., Silva, L.I.C., \& Dell'Aglio, D.D. (2014). Fatores de risco e proteção na escola: Reprovação e expectativas de futuro de jovens paraenses. Psicologia Escolar e Educacional, 18(2), 203-210.

Oliveira, M.B. \& Soares, A.B. (2011). Auto-eficácia, raciocínio verbal e desempenho escolar em estudantes. Psicologia: Teoria e Pesquisa, 27(1), 33-39.

Pacheco, J.T.B., Irigaray, T.Q., Werlang, B., Nunes, M.L.T., \& Argimon, I.R.L. (2014). Childhood maltreatment and psychological adjustment: A systematic review. Psicologia: Reflexão \& Crítica, 27(4), 815-824.

Pasian, M.S., Bazon, M.R., Pasian, S.R., \& Lacharité, C. (2014). Negligência infantil a partir do Child Neglect Index aplicado no Brasil. Psicologia: Reflexão \& Crítica, 28(1), 106-115.

Roorda, D.L. \& Koomen, H.M.Y. (2011). The influence of affective teacher-student relationships on students' school engagement and achievement: A meta-analytic approach. Review of Educational Research, 81(4), 493-529.

Schwarzer R. \& Jerusalem M. (1995). Generalized Self-Efficacy Scale. Em J. Weinman, S. Wright, \& M. Johnston (Orgs.), Measures in health psychology: A user's portfolio. Causal and control beliefs (pp. 35-37). Windsor, UK: NFER-NELSON.

Soares, A.B., Poubel, L.N., \& Mello, T.V.S. (2009). Habilidades sociais e adaptação acadêmica: Um estudo comparativo em instituições de ensino público e privado. Aletheia, 29(1), 27-42.

Squassioni, C.E. \& Matsukura, T. S. (2014). Adaptação transcultural da versão portuguesa do Social Support Appraisals para o Brasil. Psicologia: Reflexão \& Crítica, 27(1), 71-80.

Sterrett, E.M., Jones, D.J., McKee, L.G., \& Kincaid, C. (2011). Supportive non-parental adults and adolescent psychosocial functioning: Using social support as a theoretical framework. American Journal of Community Psychoogy, 48(1), 284-295.

Vaux, A., Philips, J., Holly, L., Thompson, B., Williams, D., \& Stewart, D. (1986). The Social Support Appraisals (SSA) scale: Studies of 
reliability and validity. American Journal of Community Psychology, 14, 195-220.

Yunes, M.A.M., Pietro, A.T., Silveira, S.B., Juliano, M.C.C., Garcia, N.M. (2015). Um educador para proteger do risco e tricotar a resiliência: O profissional da educação como agente de proteção e de promoção de resiliência. Em S. Cabral \& B. Cyrulnik (Orgs.), Resiliência: Como tirar leite de pedra (pp. 155-170). Casa do Psicólogo: São Paulo.
Zolkoski, S.M. \& Bullock, L.M. (2012). Resilience in children and youth: A review. Children and Youth Services Review Journal, 34(1), 2295-2303.

Zuffiano, A., Alessandri, G., Gerbino, M., Kanacri, B.P.L., Di Giunta, L., Milioni, M., \& Caprara, G.V. (2013). Academic achievement: The unique contribution of self-efficacy beliefs in self-regulated learning beyond intelligence, personality traits, and self-esteem. Learning and Individual Differences, 23(1), 158-162.

\section{Sobre as autoras}

Ana Maria Nunes El Achkar (anaelachkar@yahoo.com.br).

Doutora em Psicologia pelo Programa de Pós-graduação em Psicologia Social da Universidade Salgado de Oliveira - UNIVERSO, Niterói, RJ. Coordenadora de Pós-Graduação em Psicopedagogia Clínica e Institucional e Graduação em Pedagogia da Faculdade CNEC Itaboraí FACNEC, RJ.

Vanessa Barbosa Romera Leme (vanessaromera@gmail.com).

Doutora em Psicologia pela FFCLRP-USP. Professora no Programa de Pós-Graduação em Psicologia Social na Universidade do Estado do Rio de Janeiro - UERJ.

Adriana Benevides Soares (adribenevides@gmail.com).

Doutora em Psicologia Cognitiva pela Universidade de Paris XI. Professora do Programa de Pós-Graduação em Psicologia Social na Universidade Salgado de Oliveira - UNIVERSO, Niterói, RJ e professora Associada da Universidade do Estado do Rio de Janeiro, UERJ, Rio de Janeiro - RJ

Maria Angela Mattar Yunes (mamyunes@yahoo.com.br).

Doutora em Educação pela Pontifícia Universidade Católica de São Paulo. Professora Associada no Programa de Pós-Graduação em Educação na Universidade La Salle - Unilasalle, Canoas, RS e no Programa de Pós-Graduação em Psicologia Social da Universidade Salgado de Oliveira UNIVERSO, Niterói, RJ.

Pesquisa financiada pelo Programa de Suporte à Pós-Graduação de Instituições de Ensino Particulares - PROSUP, financiado pela Coordenação de Aperfeiçoamento de Pessoas de Nível Superior - CAPES 